\title{
Evolution of commensal bacteria in the intestinal tract of mice
}

\author{
Ana Sousa ${ }^{1,2}$, Nelson Frazão ${ }^{1}$, Ricardo S. Ramiro ${ }^{1}$ and Isabel Gordo ${ }^{1}$ \\ Addresses \\ ${ }^{1}$ Instituto Gulbenkian de Ciência, Rua da Quinta Grande, 6, Oeiras, Portugal \\ 2 iBiMED, Institute for Biomedicine, Universidade de Aveiro, Portugal \\ Corresponding author: Gordo, Isabel (igordo@igc.gulbenkian.pt)
}

\section{Highlights}

- The composition of the gut microbiota is shaped by positive and negative microbemicrobe and microbe-host interactions.

- Evolution of a bacterial species in these complex ecosystems can be very rapid.

- Natural selection overwhelms genetic drift in structuring the genetic composition of new emerging strains.

- Experimental evolution combined with high-throughput sequencing is a powerful methodology to unravel the repeatability of evolutionary change in the gut and how it is influenced by diet and host genetics.

Hundreds of different bacterial species inhabit our intestines and contribute to our health status, with significant loss of species diversity typically observed in disease conditions. Within each microbial species a great deal of diversity is hidden and such intra-specific variation is also key to the proper homeostasis between the host and its microbial inhabitants. Indeed, it is at this level that new mechanisms of antibiotic resistance emerge and pathogenic characteristics evolve. Yet, our knowledge on intra-species variation in the gut is still limited and an understanding of the evolutionary mechanisms acting on it is extremely reduced. Here we review recent work that has begun to reveal that adaptation of commensal bacteria to the mammalian intestine may be fast and highly repeatable, and that the time scales of evolutionary and ecological change can be very similar in these ecosystems. 


\section{Introduction}

"The dependence of the intestinal microbes on the food makes it possible to adopt measures to modify the flora in our bodies and to replace the harmful microbes by useful microbes" (Élie Metchnikoff, The prolongation of life, 1910, pg 162) [1]

Microbes find shelter and resources inside the guts of their hosts. Hosts find genes, new traits and functions in the microbes they harbor. In humans, it is currently estimated that for each host cell there is at least one microbial cell [2], and that the number of microbial genes is greater than the number of host genes. Highthroughput sequencing, particularly the use of 16S rRNA sequencing, has allowed for an unprecedented characterization of the gut microbiome, revealing that its composition is highly dynamic, both spatially and temporally. Starting at birth, microbes colonize hosts in a process involving ecological succession of species [3], which is characterized by large fluctuations in abundances and a high level of interhost variation. Reaching adulthood the microbial species composition becomes more stable both within and between hosts. Importantly, the composition of the gut microbiota modulates the host's ability to resist pathogens [4] and its immune homeostasis $[5,6]$. Moreover, the gut microbiota has multiple effects on peripheral organs, ranging from bile acid metabolism in the liver [7] to modulating behavior by affecting gene expression in the brain [8].

Recent advances in our understanding of gut microbial ecology and its relation to host health have been made. However, much less is known about evolutionary processes in the gut. Here we review work on how quickly and by what mechanisms evolutionary change may occur within a given bacterial species colonizing the intestine. We focus on mice to dissect key processes of microbe-microbe and hostmicrobe interactions, due to the accumulated knowledge of its physiology, genetics and behavior as a classical model organism. Furthermore, mice allow study of adaptation in the complex gut ecosystem under controlled conditions (e.g. migration, diet, temperature). Such control allows unraveling the reproducibility of the adaptation pattern in the gut, in conditions where the bacteria do not cause disease to the host. Evolution of commensal bacteria has received far less attention than the adaptation of pathogens. Nevertheless, it is important to study the evolution of commensals as this may be quite distinct from pathogens; for e.g. the fitness landscape of a pathogen may be marked by strong selection to avoid the host 
immune system and to increase adaptation to a novel environment. Another distinctive property of a pathogen's fitness landscape is a reduced number of interactions with other microbial species.

\section{Richness of interactions in the gut}

As a home for microbes, the host intestine constitutes an environment where commensal bacteria experience multiple selective pressures. Healthy hosts typically maintain a rich and stable microbiota, yet how they do so is still as mysterious as it was in the time of Metchnikoff, who believed that a long, healthy life depended on the quality of the intestinal microbes [1]. As we review below, controlled experiments in mice and developments in mathematical modeling have recently been done to help determining key interactions that shape the temporal composition of the gut microbiota and its stability.

A key component of the environment of a given microbe is another microbe, thus microbe-microbe interactions are expected to be important in the gut. To understand the nature of such interactions, ingenious experiments where a stable microbiota ecosystem is perturbed and followed through time, have been performed in mice $[9,10]$. As antibiotics cause considerable changes in the gut bacterial composition [11], they can be used as perturbations to obtain detailed temporal series data of microbiota composition (through 16S rRNA sequencing) as this recovers from the perturbation and achieves a new state of equilibrium. Assuming that a Lotka-Volterra Model (from classical ecological theory) governs microbe-microbe interactions and their dynamics, such data allows obtaining quantitative estimates of ecological interactions between groups of bacteria. Stein et al $[9,10]$ were pioneers in this integrated design (Figure 1) to estimate interaction networks in the gut. Their analysis suggests that a network of negative and positive interactions underlies the gut microbiota composition. A similar conclusion was reached in a study where mice devoid of microbes (germ-free, GF) were colonized with the cecal contents of a conventionally-raised mouse. Following a great amount of within and between mice initial variation in community dynamics, a stable microbiota composition was achieved after three weeks. Importantly, the study revealed that among 136 possible pairwise interactions between the microbes, 67\% were competitive (-/-), 16\% parasitic (+/-), 12.5\% ammensalistic (-/0), 3\% commensal $(+/ 0), 1.5 \%$ neutral $(0 / 0)$ and not a single one involved mutualism $(+/+)$ [12]. A possible consequence of intense competition may be to promote stability of the microbiota. Recent theory addressing the type of interactions underling the stability of a multispecies 
ecosystem of microbes, whose dynamics follow a Lotka-Volterra Model, predicts that many species are likely to stably coexist when the system is dominated by competition (negative pairwise interactions) [13]. We note that the Lotka-Volterra Model in addition to only considering pairwise interactions, also does not make explicit the precise mechanisms driving the interactions (such as possible metabolites that the species may exchange or specific limiting resources that may underlie competition between species) [14]. Such simplicity can have major drawbacks: e.g. previous experiments have shown that for the simplest case where two bacterial strains grow in a chemostat with a single limiting resource, the LotkaVolterra Model fails to make correct predictions on the dynamics of competition [15]. Thus, future work is needed to better understand the nature of microbe-microbe interactions in the gut.

Host-nutrition and its gut microbes are also expected to interact, as the gut microbiota is known to be important for host digestion (e.g. breakdown of complex carbohydrates). Several studies have demonstrated shifts in microbiota composition following dietary changes $[16,17]$. One example is the change from a low-fat/highpolysaccharide to a high-fat/high-sugar diet, with the latter diet leading to a strong increase in the proportion of Firmicutes relative to the Bacteroidetes phylum [17]. Importantly, a recent study has also shown that changes in diet can have transgenerational consequences for microbiota composition, with a low-fiber diet having a cumulative effect (across generations) that led to species loss. These missing microbial species could only be regained through fecal transplants from mice that had been fed with a high-fiber diet [18].

Interactions between the host immune system (IS) and gut commensals have also gained relevance. Both innate and adaptive immune responses have been shown to shape microbiota composition and determine the boundaries between the host and its microbes [19,20]. Reciprocally, commensals shape the host innate and adaptive immune responses, e.g. GF mice have reduced levels of antimicrobial peptides [21] and colonic $\mathrm{T}$ regulatory cells [22], the latter being increased by the presence of specific microbes. Regarding the innate arm of the IS, some host antimicrobial peptides were shown to cause large fitness reductions to commensal strains of Proteobacteria, but not to strains of Bacteroidetes and Firmicutes, the latter belonging to a phylum typically found at high abundances in the gut [23]. Regarding adaptive IS, host immunoglobulins A ( $\lg A s)$ were found to have diametrically opposed consequences for the fitness of different commensals. IgAs were shown to 
suppress the expansion of segmented filamentous bacteria [24], but also to promote the maintenance and diversification of certain Clostridia [5]. These examples highlight the contribution of the host to generate and maintain a diverse gut microbiota.

\section{Evolutionary change within species of the gut microbiota}

Notwithstanding the ecological interactions mentioned above, the current vision of gut microbiota composition typically ignores an important characteristic of many bacterial strains: their capacity to rapidly evolve, either by accumulating new adaptive mutations or by acquiring new genes (Figure 1). The gut microbiota should be a prime example of a system in which the ecological and evolutionary time scales may meet. The rate of evolutionary change depends primarily on population size, mutation rate and the effects on fitness of the mutations that spontaneously occur. The latter are determined by the strength of selection experienced in a given environment. Neutral mutations fix at the rate at which they emerge [25]. Deleterious mutations get eliminated, except if their effects are very small compared to the population size or if they hitchhike with beneficial variants to which they are linked. Advantageous mutations sweep to fixation with a probability proportional to their benefit [26]. In the gut, many bacterial species have population sizes composed of millions of cells, which are rapidly dividing to withstand the continuous flushing out of the intestine $[27,28]$. Given the strong and diverse selective pressures described in the previous section and typical estimates of bacterial genomic mutation rates $[29,30]$, a considerable amount of evolutionary change may be expected to occur.

Most of our understanding of bacterial evolution and adaptation has been gathered in vitro, under specific selective pressures (forward study of evolution), or by sampling extant genomes and inferring the processes that caused the observed differences (backward study of evolution). Ideally, and in the absence of a time machine, the forward and backward methods should be sufficiently complementary to allow an understanding of how bacteria evolve in nature. Remarkably, the power of experimental evolution (EE) to study evolution "in real time" has been underexplored to better understand host-microbe associations [31]. In the context of the microbiota, we believe this approach can be very helpful in answering important questions such as: How fast do commensal gut bacteria evolve? Is strain diversification driven by selection or mostly the result of a neutral process? What is the typical effect of a new emerging mutation or a gene acquired by horizontal gene transfer?

\section{Mutation and intense clonal competition in the gut}


Microbes have been key to demonstrate the power of natural selection, especially in the context of disease (e.g. evolution of drug resistance). In a healthy mammalian gut, under homeostasis, the action of natural selection on strain diversity has been less studied. While a simple assumption would be that commensal gut bacteria could be seating on a Fisherian fitness peak, and little adaptation should occur, the complexity of the gut environment, and the variation of gut microbiota composition across hosts and along time, points otherwise. There, selection may be influenced by spatial heterogeneity (e.g. nutritional, oxygen, $\mathrm{pH}$, bile salts and other gradients), environmental variation (e.g. diet changes), tradeoffs within a given host [32] and when transmitting across species [33], phage predation [34], specific host and bacterial genetic backgrounds [35,36], and migration from the external environment [37]. EE has proven important for studying the relative role of mutation, selection and drift in bacteria colonizing the gut. Using the simplest possible system, a GF host (mice) that is then colonized with a single bacterial species, Giraud et al [38] observed the independent emergence of strains with high mutation rates (mutators). Mutators could spread due to their increased ability to rapidly generate new adaptive variants during colonization of GF mice. In another colonization study, but now with conventional mice which have a complex gut microbiota, Barroso-Batista et al [39] followed the sequential accumulation of beneficial mutations in two fluorescentlylabeled isogenic $E$. coli lineages sampled from feces. The timing and change in frequency of the fluorescences, caused by the emergence of new alleles, allowed the authors to estimate a rate of adaptive mutations of $\sim 7 \times 10^{-7}$ per generation, with a mean effect of $\sim 7 \%$. Importantly, multiple clones, carrying mutations at different loci, emerged and competed during adaptation to the mouse gut in a process known as clonal interference (Figure 2). The strong fitness effects of the emerging mutations in a given host (ranging from $2 \%$ to $14 \%$ [40]) were further shown to provide equivalent benefits in new hosts, suggesting that $E$. coli experiences similar pressures when colonizing genetically identical mice that eat the same food. Consistent with this interpretation, the molecular path of evolution taken by the bacteria, when colonizing different hosts, was extremely similar - evolutionary parallelism [39,40]. Moreover, an average of $\sim 2$ mutations per genome were found to accumulate after $\sim 450$ generations (24 days). These results indicate that the tempo and mode of short-term molecular evolution can be highly repeatable in a complex gut ecosystem. In a longer-term EE, using a different $E$. coli strain colonizing outbred mice with a complex microbiota, Lescat et al [41] found that after about a year (> 6500 generations), 6.3 mutations per clone had accumulated. Moreover, they also 
observed substantial levels of parallel molecular evolution, arguing for a major role for selection in the process of strain diversification.

In both the short- and the long-term E. coli colonization studies, some level of epistasis between the accumulated mutations was observed [40,41], suggesting that the genetic basis of gut adaptation can be conditioned by the strain's genetic background. This highlights not only the need to study other strains, but also to determine if the typical rate of evolution in true mouse commensal strains is as high as for the strains followed in these studies, which were originally isolated from human stool.

Host genetics have also been shown to affect the tempo and mode of adaptation of these bacteria to the mouse gut. When comparing wild-type with Rag2-/- mice, (which lack $B$ and $T$ cells and therefore are severely immunocompromised) BarrosoBatista et al [35] found that the dynamics of E. coli gut adaptation were slower and the effects of the emerging mutations more variable, an effect attributed to the different microbiota compositions in the two host genotypes.

EE also allows understanding the selective pressures experienced by bacteria in the gut environment. Nutritional optimization was found to be one of the main challenges bacteria face in the microbiota community. Mutants that can grow faster on sugars present in the mucus $[42,43]$ and specialist clones $[44,45]$ that are able to explore different niches have been shown to emerge during adaptation to the mouse gut. In accordance with these findings, several studies $[46,47]$ exploring the mechanisms by which an "uncompromised microbiota prevents pathogen infections" (colonization resistance, [48]) support the hypothesis that strains with completely overlapping nutritional niches might not be able to co-exist in the same community. This is the basis of the 'nutrient-niche hypothesis', first enunciated by Freter (reviewed in [49]), which postulates that microbes can only persist in a complex community if they use at least one limiting nutrient better than all others. This hypothesis assumes that bacteria compete for a nutrient pool that is equally available throughout the gut. To account for the inherent spatial structure and species distribution in the gut, the 'restaurant hypothesis' was developed (reviewed in [36]). This states that the potential for long-term colonization by facultative anaerobes (such as $E$. coli and Salmonella) depends on their ability to acquire nutrients locally in mixed-species biofilms. This hypothesis, coupled with the results of evolution experiments showing fast strain diversification, possibly driven by competition for limiting nutrients, suggests that bacterial nutritional adaptation may alter colonization resistance.

\section{Horizontal gene transfer in the gut}


Bacterial strain diversification can result from horizontal gene transfer (HGT), which may occur among distantly-related bacteria or even inter-kingdom species [50-53]. However, its efficiency was shown to decrease exponentially with sequence divergence [54,55]. HGT takes place via three different mechanisms: conjugationmediated plasmid exchange, phage-mediated transduction or natural transformation [56]. Given its high bacterial density, the mammalian gut is likely a hotspot for HGT when compared to other ecosystems [54,57]. In humans, the gut is predicted to be the body site with the highest number of horizontally acquired genes per microbe (average of 48.6 genes) [58]. Horizontally-acquired genetic material, which can constitute up to $20 \%$ of prokaryotic genomes [59-61], enables a quantum leap in gene diversification of particular members of the gut microbiota, potentially changing their own evolutionary fate and that of the whole community.

Important questions concerning the role of HGT in the evolution of gut microbes remain unanswered: What is the typical rate of each HGT mechanism in the gut? What is the typical fitness effect of an HGT event? The mouse as model of bacterial colonization has been used to elucidate these issues.

Conjugation within the microbiota can be common and occur in a short timeframe. A study using GF mice, colonized with human feces, found that a natural plasmid could be transferred between $E$. coli strains at a frequency of $10^{-5}$ after 6 hours of colonization [62]. In another study, the in vivo conjugation frequency of transposon Tn1545 from Enterococcus faecalis to Listeria monocytogenes in the gut of gnotobiotic mice was $1.1 \times 10^{-8}$ after 35 days [50]. In the context of gut inflammation, Stecher et al [63] observed that blooms of infecting Salmonella cells and of resident commensal $E$. coli lead to extremely high rates of conjugation of the colicin-plasmid P2 (plasmid present in all E. coli cells within 4 days).

Although metagenomic data show that bacteria from the gut microbiota carry considerable numbers of temperate phages [64], direct measurements of the rate of phage-mediated HGT (transduction) within the mammalian intestine have been understudied. Using the classical system of $E$. coli and its best studied phage $\lambda$, De Paepe et al [65] have undertaken a well-designed quantitative study to determine the rate of prophage induction and the fitness effects of prophage integration into the bacteria colonizing the gut of GF mice. They showed that the gut is an excellent environment for phage to spread, and estimated a rate of prophage induction of $\sim 2 \%$ per generation, which is much higher than observed in laboratory conditions. Such 
high induction was also shown to cause considerable fitness costs to the infected bacteria.

Finally, transformation was observed to be common in the respiratory tract, where the naturally competent Streptococcus pneumoniae shows a transformation frequency of $10^{-2}$ after 2 days of colonization [66]. However, transformation appears to be relatively rare in the mammalian gut. This may be due to a low amount of free DNA for transformation, either due to DNA shielding by the gut contents [67] or to DNA-degrading enzymes [63]. More studies will be required to accurately assess transformation rates in the gut.

Importantly, the horizontally-transferred traits that are under selection as bacteria adapt in the mouse gut are poorly known. Modi et al [64] found that, in addition to antibiotic-resistance genes, phage genomes are also enriched for multiple genes related to metabolism (e.g. carbohydrate metabolism or glycan synthesis and metabolism) after antibiotic treatment. In agreement with what was found in EE studies (previous section), these data could suggest that nutritional adaptation is one of the key selective pressures in the mouse gut and that both mutational and HGT processes contribute to that adaptation.

\section{Conclusions}

The extent to which evolutionary change occurs in the gut and shapes the genetic structure of its microbiota is still largely unknown. The findings stemming from the few studies of evolution in mice suggest that a bacterial population in the gut could contain several evolving clones, differing by several mutations or horizontallyacquired gene(s) with high selective effects. If these observations turn out to be general, two implications emerge: i) a complete account of the genetic diversity within microbial ecosystems inhabiting a host could be a difficult task, as intraspecies variation may be both large and highly dynamic [65]; ii) the strong selection for nutritional optimization indicates it may be "possible to adopt measures to modify the flora in our bodies" [1], by precisely manipulating diet to control which evolving strains may be allowed to stay in the gut.

Rapid evolutionary change can be critical to community structure [66], and to the observed diversity of the mammalian microbiota. Future theory should therefore consider whether incorporating high adaptive mutation or HGT event rates into 
classical models of ecology may help explain one of the big mysteries of nature: how can large numbers of species be maintained in ecosystems?

\section{Acknowledgement}

We thank the Deutsche Forschungsgemeinschaft Grant SFB 680 and the Portuguese Science and Technology Foundation FCT (SFRH/BPD/111725/2015 and UID/BIM/04501/2013 support to AS, SFRH/BPD/110750/2015 support to NF). The funders had no role in study design, decision to publish or preparation of the manuscript.

Figure 1. Inference of microbe-microbe ecological interactions from time-series data after perturbation of the gut microbiota ecosystem.

The changes in relative abundance of operational taxonomic units (OTUs) over time after perturbation (such as diet alteration or antibiotics) allow estimation of ecological interactions. Here we represent a hypothetical example of a community dynamics returning to equilibrium. After an initial perturbation (indicated in 1), where the relative abundances suffer major alterations, the community stabilizes from day 35 onwards. The composition of the microbiota can be determined through 16S rRNA or whole metagenomic analysis of fecal material (indicated in 2). Following the dynamics upon perturbation and through the recovery period, it is possible to obtain a matrix of ecological interactions among the different OTUs (genus/family), under a LotkaVolterra model as in $[9,10]$. In this matrix (indicated in 3 ), blue shading represents negative interactions, yellow positive interactions and white lack of interaction; the intensity of the color is proportional to the strength of the interaction. This methodology ignores the hidden strain variation (including de novo emerging strains). These new variants differ from each other in at least one mutation (duplication, deletion, gene acquisition, gene inactivation and SNP; (indicated in 4)). Using a similar method as in $[9,10]$, it should be possible to estimate the network of interactions between strains. 
Figure 2. Evolutionary dynamics of de novo emerging strains: multiple adaptive mutations compete for increasing in frequency - clonal interferenceduring $E$. coli colonization of the mouse gut.

Examples of the emergence of strain variation within a lineage of $E$. coli colonizing two hosts (adapted from Barroso-Batista et al [39]). Muller plots where new adaptive mutations spread in an initial isogenic population of fluorescently-labeled $E$. coli (either blue or yellow), which was used to colonize the intestine of streptomycintreated mice. A, Y, Z and C represent genes from the galactitol operon, whose inactivation was shown to be adaptive. Each of the distinct alleles is equally fit in the gut and therefore polymorphism can be maintained for several days. srIR, $d c u B$ and focA represent secondary targets for adaptive mutations. The two Muller plots represent independent mice, showing that parallelism in the genetic targets of adaptation is extensive. The darker the tone of blue or yellow, the higher the number of mutations carried by a given clone. For example, in the right panel, the ancestral strain in the blue background first acquires a mutation in gat $A$ ( day 6$)$. By day 11 a small proportion of that population acquires a second mutation in $s r l R$, which is then followed by a third mutation in focA, thus creating a triple mutant in 24 days.

\section{References and Recommended reading}

1. Metchnikoff E, Mitchell PC: The prolongation of life; optimistic studies. New York \& London: G.P. Putnam's Sons; 1908.

2. Sender R, Fuchs S, Milo R: Are We Really Vastly Outnumbered? Revisiting the Ratio of Bacterial to Host Cells in Humans. Cell 2016, 164:337-340.

3. Yassour M, Vatanen T, Siljander H, Hamalainen A-M, Harkonen T, Ryhanen SJ, Franzosa EA, Vlamakis H, Huttenhower C, Gevers D, et al.: Natural history of the infant gut microbiome and impact of antibiotic treatment on bacterial strain diversity and stability. Sci. Transl. Med. 2016, 8:343ra81-343ra81.

4. Buffie CG, Bucci V, Stein RR, McKenney PT, Ling L, Gobourne A, No D, Liu $H$, Kinnebrew $M$, Viale $A$, et al.: Precision microbiome reconstitution restores bile acid mediated resistance to Clostridium difficile. Nature 2014, 517:205-208.

5. Kawamoto S, Maruya M, Kato LM, Suda W, Atarashi K, Doi Y, Tsutsui Y, Qin $\mathrm{H}$, Honda K, Okada T, et al.: Foxp3+ T Cells Regulate Immunoglobulin A Selection and Facilitate Diversification of Bacterial Species Responsible for Immune Homeostasis. Immunity 2014, 41:152-165.

6. Atarashi K, Tanoue T, Oshima K, Suda W, Nagano Y, Nishikawa H, Fukuda $\mathrm{S}$, Saito T, Narushima S, Hase $\mathrm{K}$, et al.: Treg induction by a rationally 
selected mixture of Clostridia strains from the human microbiota. Nature 2013, 500:232-236.

7. Sayin SI, Wahlström A, Felin J, Jäntti S, Marschall H-U, Bamberg K, Angelin B, Hyötyläinen T, Orešič M, Bäckhed F: Gut Microbiota Regulates Bile Acid Metabolism by Reducing the Levels of Tauro-beta-muricholic Acid, a Naturally Occurring FXR Antagonist. Cell Metab. 2013, 17:225-235.

8. Bravo JA, Forsythe P, Chew MV, Escaravage E, Savignac HM, Dinan TG, Bienenstock J, Cryan JF: Ingestion of Lactobacillus strain regulates emotional behavior and central GABA receptor expression in a mouse via the vagus nerve. Proc. Natl. Acad. Sci. 2011, 108:16050-16055.

9. Stein RR, Bucci V, Toussaint NC, Buffie CG, Rätsch G, Pamer EG, Sander C, Xavier JB: Ecological Modeling from Time-Series Inference: Insight into Dynamics and Stability of Intestinal Microbiota. PLoS Comput. Biol. 2013, 9:e1003388.

10. Bucci V, Tzen B, Li N, Simmons M, Tanoue T, Bogart E, Deng L, Yeliseyev V, Delaney ML, Liu Q, et al.: MDSINE: Microbial Dynamical Systems INference Engine for microbiome time-series analyses [Internet]. Genome Biol. 2016, 17.

11. Sjölund M, Wreiber K, Andersson DI, Blaser MJ, Engstrand L: Long-term persistence of resistant Enterococcus species after antibiotics to eradicate Helicobacter pylori. Ann. Intern. Med. 2003, 139:483-487.

12. Marino S, Baxter NT, Huffnagle GB, Petrosino JF, Schloss PD: Mathematical modeling of primary succession of murine intestinal microbiota. Proc. Natl. Acad. Sci. 2014, 111:439-444.

13. Coyte KZ, Schluter J, Foster KR: The ecology of the microbiome: Networks, competition, and stability. Science 2015, 350:663-666.

14. Momeni B, Xie L, Shou W: Lotka-Volterra pairwise modeling fails to capture diverse pairwise microbial interactions [Internet]. eLife 2017, 6.

15. Hansen SR, Hubbell SP: Single-nutrient microbial competition: qualitative agreement between experimental and theoretically forecast outcomes. Science 1980, 207:1491-1493.

16. David LA, Maurice CF, Carmody RN, Gootenberg DB, Button JE, Wolfe BE, Ling AV, Devlin AS, Varma Y, Fischbach MA, et al.: Diet rapidly and reproducibly alters the human gut microbiome. Nature 2013, 505:559563.

17. Turnbaugh PJ, Bäckhed F, Fulton L, Gordon JI: Diet-Induced Obesity Is Linked to Marked but Reversible Alterations in the Mouse Distal Gut Microbiome. Cell Host Microbe 2008, 3:213-223.

18. Sonnenburg ED, Smits SA, Tikhonov M, Higginbottom SK, Wingreen NS, Sonnenburg JL: Diet-induced extinctions in the gut microbiota compound over generations. Nature 2016, 529:212-215.

19. Slack E, Hapfelmeier S, Stecher B, Velykoredko Y, Stoel M, Lawson MAE, Geuking MB, Beutler B, Tedder TF, Hardt W-D, et al.: Innate and Adaptive Immunity Cooperate Flexibly to Maintain Host-Microbiota Mutualism. Science 2009, 325:617-620.

20. Maynard CL, Elson CO, Hatton RD, Weaver CT: Reciprocal interactions of the intestinal microbiota and immune system. Nature 2012, 489:231-241.

21. Vaishnava S, Behrendt CL, Ismail AS, Eckmann L, Hooper LV: Paneth cells directly sense gut commensals and maintain homeostasis at the intestinal host-microbial interface. Proc. Natl. Acad. Sci. 2008, 105:2085820863.

22. Faith JJ, Ahern PP, Ridaura VK, Cheng J, Gordon JI: Identifying Gut Microbe-Host Phenotype Relationships Using Combinatorial Communities in Gnotobiotic Mice. Sci. Transl. Med. 2014, 6:220ra11220 ra11. 
23. Cullen TW, Schofield WB, Barry NA, Putnam EE, Rundell EA, Trent MS, Degnan PH, Booth CJ, Yu H, Goodman AL: Antimicrobial peptide resistance mediates resilience of prominent gut commensals during inflammation. Science 2015, 347:170-175.

24. Suzuki K, Meek B, Doi Y, Muramatsu M, Chiba T, Honjo T, Fagarasan S: Aberrant expansion of segmented filamentous bacteria in IgA-deficient gut. Proc. Natl. Acad. Sci. 2004, 101:1981-1986.

25. Birky CW, Walsh JB: Effects of linkage on rates of molecular evolution. Proc. Natl. Acad. Sci. U. S. A. 1988, 85:6414-6418.

26. Gillespie JH: Population genetics: a concise guide. Johns Hopkins University Press; 2004.

27. Rang CU, Licht TR, Midtvedt T, Conway PL, Chao L, Krogfelt KA, Cohen PS, Molin S: Estimation of growth rates of Escherichia coli BJ4 in streptomycin-treated and previously germfree mice by in situ rRNA hybridization. Clin. Diagn. Lab. Immunol. 1999, 6:434-436.

28. Myhrvold C, Kotula JW, Hicks WM, Conway NJ, Silver PA: A distributed cell division counter reveals growth dynamics in the gut microbiota. Nat. Commun. 2015, 6:10039.

29. Drake JW: A constant rate of spontaneous mutation in DNA-based microbes. Proc. Natl. Acad. Sci. U. S. A. 1991, 88:7160-7164.

30. Wielgoss S, Barrick JE, Tenaillon O, Cruveiller S, Chane-Woon-Ming B, Medigue C, Lenski RE, Schneider D, Andrews BJ: Mutation Rate Inferred From Synonymous Substitutions in a Long-Term Evolution Experiment With Escherichia coli. G358 GenesGenomesGenetics 2011, 1:183-186.

31. Hoang KL, Morran LT, Gerardo NM: Experimental Evolution as an Underutilized Tool for Studying Beneficial Animal-Microbe Interactions [Internet]. Front. Microbiol. 2016, 7.

32. De Paepe M, Gaboriau-Routhiau V, Rainteau D, Rakotobe S, Taddei F, CerfBensussan N: Trade-Off between Bile Resistance and Nutritional Competence Drives Escherichia coli Diversification in the Mouse Gut. PLoS Genet. 2011, 7:e1002107.

33. Frese SA, Benson AK, Tannock GW, Loach DM, Kim J, Zhang M, Oh PL, Heng NCK, Patil PB, Juge N, et al.: The Evolution of Host Specialization in the Vertebrate Gut Symbiont Lactobacillus reuteri. PLoS Genet. 2011, 7:e1001314.

34. Reyes A, Wu M, McNulty NP, Rohwer FL, Gordon JI: Gnotobiotic mouse model of phage-bacterial host dynamics in the human gut. Proc. Natl. Acad. Sci. 2013, 110:20236-20241.

35. Barroso-Batista J, Demengeot J, Gordo I: Adaptive immunity increases the pace and predictability of evolutionary change in commensal gut bacteria. Nat. Commun. 2015, 6:8945.

36. Conway T, Cohen PS: Commensal and Pathogenic Escherichia coli Metabolism in the Gut [Internet]. In Metabolism and Bacterial Pathogenesis. Edited by Conway T, Cohen PS. American Society of Microbiology; 2015:343-362.

37. Seedorf H, Griffin NW, Ridaura VK, Reyes A, Cheng J, Rey FE, Smith MI, Simon GM, Scheffrahn RH, Woebken D, et al.: Bacteria from Diverse Habitats Colonize and Compete in the Mouse Gut. Cell 2014, 159:253266.

38. Giraud A: Costs and Benefits of High Mutation Rates: Adaptive Evolution of Bacteria in the Mouse Gut. Science 2001, 291:2606-2608.

39. Barroso-Batista J, Sousa A, Lourenço M, Bergman M-L, Sobral D, Demengeot J, Xavier KB, Gordo I: The First Steps of Adaptation of Escherichia coli to the Gut Are Dominated by Soft Sweeps. PLoS Genet. 2014, 10:e1004182. 
40. Lourenço M, Ramiro RS, Güleresi D, Barroso-Batista J, Xavier KB, Gordo I, Sousa A: A Mutational Hotspot and Strong Selection Contribute to the Order of Mutations Selected for during Escherichia coli Adaptation to the Gut. PLOS Genet. 2016, 12:e1006420.

41. Lescat M, Launay A, Ghalayini M, Magnan M, Glodt J, Pintard C, Dion S, Denamur $E$, Tenaillon $O$ : Using long-term experimental evolution to uncover the patterns and determinants of molecular evolution of an Escherichia coli natural isolate in the streptomycin-treated mouse gut [Internet]. Mol. Ecol. 2016, doi:10.1111/mec.13851.

42. Leatham MP, Stevenson SJ, Gauger EJ, Krogfelt KA, Lins JJ, Haddock TL, Autieri SM, Conway T, Cohen PS: Mouse Intestine Selects Nonmotile flhDC Mutants of Escherichia coli MG1655 with Increased Colonizing Ability and Better Utilization of Carbon Sources. Infect. Immun. 2005, 73:8039-8049.

43. Fabich AJ, Leatham MP, Grissom JE, Wiley G, Lai H, Najar F, Roe BA, Cohen PS, Conway T: Genotype and Phenotypes of an Intestine-Adapted Escherichia coli K-12 Mutant Selected by Animal Passage for Superior Colonization. Infect. Immun. 2011, 79:2430-2439.

44. Leatham-Jensen MP, Frimodt-Moller J, Adediran J, Mokszycki ME, Banner ME, Caughron JE, Krogfelt KA, Conway T, Cohen PS: The StreptomycinTreated Mouse Intestine Selects Escherichia coli envZ Missense Mutants That Interact with Dense and Diverse Intestinal Microbiota. Infect. Immun. 2012, 80:1716-1727.

45. Sousa A, Ramiro RS, Barroso-Batista J, Güleresi D, Lourenço M, Gordo I: Rampant Reverse Evolution towards Specialization in Commensal Bacteria Colonizing the Gut [Internet]. 2016, [no volume].

46. Leatham MP, Banerjee S, Autieri SM, Mercado-Lubo R, Conway T, Cohen PS: Precolonized Human Commensal Escherichia coli Strains Serve as a Barrier to E. coli 0157:H7 Growth in the Streptomycin-Treated Mouse Intestine. Infect. Immun. 2009, 77:2876-2886.

47. Freter R, Brickner H, Fekete J, Vickerman MM, Carey KE: Survival and Implantation of Escherichia coli in the Intestinal Tract. Infect. Immun. 1983, [no volume].

48. Stecher B, Berry D, Loy A: Colonization resistance and microbial ecophysiology: using gnotobiotic mouse models and single-cell technology to explore the intestinal jungle. FEMS Microbiol. Rev. 2013, 37:793-829.

49. Freter R: Factors affecting the microecology of the gut [Internet]. In Probiotics. . Springer Netherlands; 1992:111-144.

50. Doucet-Populaire F, Trieu-Cuot P, Dosbaa I, Andremont A, Courvalin P: Inducible transfer of conjugative transposon Tn1545 from Enterococcus faecalis to Listeria monocytogenes in the digestive tracts of gnotobiotic mice. Antimicrob. Agents Chemother. 1991, 35:185-187.

51. Jones BV, Sun F, Marchesi JR: Comparative metagenomic analysis of plasmid encoded functions in the human gut microbiome. $B M C$ Genomics 2010, 11:46.

52. Dagan T, Artzy-Randrup $Y$, Martin W: Modular networks and cumulative impact of lateral transfer in prokaryote genome evolution. Proc. Natl. Acad. Sci. 2008, 105:10039-10044.

53. Llosa M, Schröder G, Dehio C: New perspectives into bacterial DNA transfer to human cells. Trends Microbiol. 2012, 20:355-359.

54. Smillie CS, Smith MB, Friedman J, Cordero OX, David LA, Alm EJ: Ecology drives a global network of gene exchange connecting the human microbiome. Nature 2011, 480:241-244. 
55. Vulić M, Dionisio F, Taddei F, Radman M: Molecular keys to speciation: DNA polymorphism and the control of genetic exchange in enterobacteria. Proc. Natl. Acad. Sci. U. S. A. 1997, 94:9763-9767.

56. Narra HP, Ochman H: Of What Use Is Sex to Bacteria? Curr. Biol. 2006, 16:R705-R710.

57. Kelly BG, Vespermann A, Bolton DJ: Gene transfer events and their occurrence in selected environments. Food Chem. Toxicol. 2009, 47:978983.

58. Liu L, Chen X, Skogerbø G, Zhang P, Chen R, He S, Huang D-W: The human microbiome: A hot spot of microbial horizontal gene transfer. Genomics 2012, 100:265-270.

59. Garcia-Vallvé S, Romeu A, Palau J: Horizontal gene transfer in bacterial and archaeal complete genomes. Genome Res. 2000, 10:1719-1725.

60. Lawrence JG, Ochman H: Amelioration of bacterial genomes: rates of change and exchange. J. Mol. Evol. 1997, 44:383-397.

61. Médigue C, Rouxel T, Vigier P, Hénaut A, Danchin A: Evidence for horizontal gene transfer in Escherichia coli speciation. J. Mol. Biol. 1991, 222:851-856.

62. Maisonneuve null, Ouriet null, Duval-Iflah null: Effects of yoghurt intake on plasmid transfer and colonisation with transconjugants in the digestive tract of mice associated with human faecal flora. FEMS Microbiol. Ecol. 2000, 31:241-248.

63. Stecher B, Denzler R, Maier L, Bernet F, Sanders MJ, Pickard DJ, Barthel M, Westendorf AM, Krogfelt KA, Walker AW, et al.: Gut inflammation can boost horizontal gene transfer between pathogenic and commensal Enterobacteriaceae. Proc. Natl. Acad. Sci. 2012, 109:1269-1274.

64. Reyes A, Haynes M, Hanson N, Angly FE, Heath AC, Rohwer F, Gordon JI: Viruses in the faecal microbiota of monozygotic twins and their mothers. Nature 2010, 466:334-338.

65. De Paepe M, Tournier L, Moncaut E, Son O, Langella P, Petit M-A: Carriage of $\lambda$ Latent Virus Is Costly for Its Bacterial Host due to Frequent Reactivation in Monoxenic Mouse Intestine. PLOS Genet. 2016, 12:e1005861.

66. Marks LR, Reddinger RM, Hakansson AP: High Levels of Genetic Recombination during Nasopharyngeal Carriage and Biofilm Formation in Streptococcus pneumoniae. mBio 2012, 3:e00200-12-e00200-12.

67. Nordgård L, Nguyen T, Midtvedt T, Benno Y, Traavik T, Nielsen KM: Lack of detectable DNA uptake by bacterial gut isolates grown in vitro and by Acinetobacter baylyi colonizing rodents in vivo. Environ. Biosafety Res. 2007, 6:149-160.

Highlighted References:

* of special interest

** of outstanding interest

* De Paepe M, Tournier L, Moncaut E, Son O, Langella P, Petit M-A: Carriage of $\lambda$ Latent Virus Is Costly for Its Bacterial Host due to Frequent Reactivation in Monoxenic Mouse Intestine. PLOS Genet. 2016, 12:e1005861 
Pioneer study quantifying the rate of transfer of temperate phages in the gut. This study also shows that the bacteria carrying temperate phages pay a fitness cost due to the high induction rate in the mouse intestine.

** Stein RR, Bucci V, Toussaint NC, Buffie CG, Rätsch G, Pamer EG, Sander C, Xavier JB: Ecological Modeling from Time-Series Inference: Insight into Dynamics and Stability of Intestinal Microbiota. PLOS Comput. Biol. 2013, 9:e1003388

Pioneer study demonstrating how an integrative design, which combines mice experiments, microbiota $16 \mathrm{~S}$ data and mathematical modeling, may allow for quantifying ecological interactions.

** Barroso-Batista J, Sousa A, Lourenço M, Bergman M-L, Sobral D, Demengeot J, Xavier KB, Gordo I: The First Steps of Adaptation of Escherichia coli to the Gut Are Dominated by Soft Sweeps. PLoS Genet. 2014, 10:e1004182.

Using the tools of experimental evolution, this study demonstrates that rapid evolutionary change and intense clonal interference can occur in commensal species colonizing the mammalian gut. This work provides the first estimates of the rate and fitness effects of beneficial mutations in this ecosystem.

* Lescat M, Launay A, Ghalayini M, Magnan M, Glodt J, Pintard C, Dion S, Denamur $E$, Tenaillon $O$ : Using long-term experimental evolution to uncover the patterns and determinants of molecular evolution of an Escherichia coli natural isolate in the streptomycin-treated mouse gut. Mol. Ecol. 2016, doi:10.1111/mec.13851.

Study of long-term evolution of a human commensal E. coli in the mouse intestine, demonstrating a pattern of evolutionary parallelism and pinpointing the role of epistasis between adaptive mutations.

* Kawamoto S, Maruya M, Kato LM, Suda W, Atarashi K, Doi Y, Tsutsui Y, Qin H, Honda K, Okada T, et al.: Foxp3+ T Cells Regulate Immunoglobulin A Selection and Facilitate Diversification of Bacterial Species Responsible for Immune Homeostasis. Immunity 2014, 41:152-165. 
Study showing that regulatory T-cells, through their effects on $\lg A$ production, lead to increased diversity of the microbiota, particularly in Clostridia (phylum Firmicutes), and that these bacteria also induce IgA production, creating a positive feedback loop between the microbiota and the host immune system.

** Coyte KZ, Schluter J, Foster KR: The ecology of the microbiome: Networks, competition, and stability. Science 2015, 350:663-666.

This study develops mathematical theory based on the Lotka-Volterra ecological model to understand the factors that promote microbiota stability. The theory predicts that ecosystem stability is promoted by increasing the proportion of competitive, relative to cooperative interactions, as it dampens the destabilizing effects of positive feedback loops arising from cooperation. 